\title{
ANESTESIA REGIONAL SUBARACNOIDEA VS. GENERAL BALANCEADA EN HISTERECTOMÍA ABDOMINAL TOTAL Estudio de corte transversal en el Hospital de San José
}

Edward Meneses MD.*, Mónica Torres MD.**, Rafael Turriago MD.***

\section{Resumen}

la histerectomía abdominal total es uno de los procedimientos quirúrgicos ginecológicos más practicados en el Hospital de San José. La discusión sobre cual es mejor entre la AG y la AR para este tipo de procedimientos, data de $1936 .{ }^{1}$ De igual forma la presentación de eventos adversos que se observan en la unidad de cuidados postanestésicos (UCPA) han sido objeto de múltiples estudios ${ }^{1,2}$ para definir cuál técnica puede ser superior, sin que hasta el momento se haya podido determinar diferencias entre las dos. Este estudio tipo corte transversal busca encontrarlas en cuanto al consumo de opioides, rescate de dolor en la UCPA y la presentación de eventos adversos.

Materiales y métodos: es un estudio de corte transversal en casos llevados a histerectomía abdominal. El tamaño de la muestra es de 29 pacientes recolectadas por muestreo intencionado, 20 recibieron AG balanceada y nueve AR subaracnoidea, siguiendo control estricto de los criterios de inclusión, estandarización de procedimientos para cada una de las técnicas, consentimiento informado y aprobación del comité de ética médica del Hospital de San José. La recolección de los datos se llevó a cabo mediante un formato diseñado para el estudio donde se incluían la medición de escalas validadas y una encuesta final. El análisis se realizó mediante el programa Epiinfo versión 2002.

el promedio de edad fue de 44 años. El 10\% de las pacientes sometidas a histerectomía abdominal total bajo AG (dos) requirió manejo farmacológico con efedrina o vasoactivos para corregir la inestabilidad hemodinámica, mientras en el grupo en el cual se empleó AR el 11\% (una) requirió manejo farmacológico. El promedio de sangrado con AG fue de $526.75 \mathrm{ml}$ mientras con AR fue de $445.55 \mathrm{ml}$. El promedio de dolor a los 30 minutos fue de 6 para AG y $5.4 \mathrm{AR}$, a los 60 minutos fue de 4.2 y 6.1 , a los 90 minutos fue de 2 y 1.6 , a los 150 minutos fue de 0 y 1.1 , y por último a los 180 minutos fue de 0 para ambas técnicas; la dosis total de morfina en promedio utilizada en el postoperatorio correspondió a $3.9 \mathrm{mg}$ con AG y $4.1 \mathrm{mg}$ con AR.

anestesia regional, anestesia general, histerectomía abdominal, dolor postoperatorio, estabilidad hemodinámica.

$\mathrm{AG}$, anestesia general; $\mathrm{AR}$, anestesia regional; UCPA, unidad de cuidados postanestésicos; $\mathrm{EV}$, endovenosa.

\footnotetext{
Fecha recibido: febrero 20 de 2007 - Fecha aceptado: marzo 12 de 2007

Jefe de anestesiología, Hospital Infantil Universitario de San José.

* Anestesióloga Hospital de San José.

*** Anestesiólogo Clínica de Marly.
} 


\section{Introducción}

La histerectomía abdominal es un procedimiento que se realiza con frecuencia en nuestra institución. Las técnicas general o espinal son buenas opciones y están descritas para este tipo de cirugía. El uso de la última disminuye la posibilidad de náuseas, vómito, vértigo y dolor postoperatorios comparados con anestesia general. ${ }^{1,2,3}$ Otro punto importante a tratar es el tiempo de recuperación y la salida de la UCPA, pues con AR la recuperación es más rápida, ${ }^{4}$ lo cual genera una disminución en costos. También se analiza el comportamiento hemodinámico (tensión arterial, frecuencia cardíaca, sangrado intraoperatorio y necesidad de manejo farmacológico para mantener estabilidad hemodinámica) con cada una de las técnicas.

En este estudio nuestro principal objetivo es encontrar diferencias en cuanto a la incidencia de dolor y consumo de opioides como rescate en la UCPA. La incidencia estimada de dolor moderado a severo en el postoperatorio es de $29,7 \% 0^{5}$ y es primordial para el anestesiólogo manejarlo en forma adecuada para disminuirlo al 5\% según el Audit Comisión. ${ }^{6}$ Según un estudio en pacientes para cirugía ortopédica la incidencia de dolor moderado a severo en la UCPA, fue mayor de manera estadísticamente significativa en el grupo con AG (61\%) vs AR (15\%) $\mathrm{p}<0.01$. $^{7}$ Sin embargo, en múltiples investigaciones los resultados son variables, pues en unos se logra disminuir el consumo de opioides con técnicas regionales ${ }^{2,8.9}$ mientras en otros la incidencia de dolor y consumo de opioides como rescate, no varían con las dos técnicas. ${ }^{10}$ En nuestro estudio de corte prospectivo se analiza la incidencia de dolor, consumo de opioides como rescate en UCPA, incidencia de vómito postoperatorio y tiempo de recuperación en las pacientes llevadas a histerectomía abdominal utilizando AG o AR.

\section{Materiales y métodos}

Se realizó un estudio descriptivo tipo corte transversal, anotando en cada caso el tipo de anestesia utilizada y el comportamiento del dolor, consumo de opioides como rescate en UCPA, vómito, estabilidad hemodinámica, sangrado y tiempos de inducción y quirúrgico. Se realizó muestreo intencionado en 29 pacientes que cumplían la totalidad de los criterios de inclusión.

Se obtuvo aprobación por parte del comité de ética médica del Hospital de San José, se incluyeron pacientes ASA I-II, entre los 18 y 60 años, para histerectomía abdominal por miomatosis uterina o hemorragia uterina, quienes estuvieron de acuerdo con el estudio. Se definieron los siguientes criterios de exclusión: patología ginecológica oncológica, histerectomía de urgencia, anestesia espinal fallida y quienes no deseaban ingresar al estudio.

El anestesiólogo encargado de cada caso fue quien decidió la técnica anestésica, de acuerdo con la paciente y su estado clínico. A todas se les colocó monitoría básica (tensiómetro, pulsooxímetro, electrocardiograma, análisis de gases, capnografía). Ambos grupos recibieron una carga de $5 \mathrm{ml} / \mathrm{k}$ de lactato de ringer por venoclisis previo a la inducción anestésica.

El grupo de AG recibió para inducción: propofol 2-4 mg/k, fentanilo $1-2 \mathrm{mcg} / \mathrm{k}$ y cisatracurio 0.15 $\mathrm{mg} / \mathrm{k}$. Se llevó a cabo intubación orotraqueal con tubo \# 7,5 en todos los casos y para mantenimiento se utilizó sevoflurano en concentración de 1 a 1,2\% y remifentanilo 0.25 a $1 \mathrm{mcg} / \mathrm{k} / \mathrm{min}$.

En el grupo de AR, previa asepsia y antisepsia de la región lumbar, se realizó punción en L2-L3 o L3-L4 con la paciente en decúbito lateral derecho. Se utilizó en todos los casos aguja tipo Quincke \# 27 por vía mediana y luego de la aspiración de liquido cefalorraquídeo se aplicó una dosis de bupivacaína al 0.5\% pesada $15 \mathrm{mg}$ obteniendo un nivel sensitivo T6.

En ambos grupos se empleó como profilaxis para el vómito postoperatorio una dosis de dexametasona de $8 \mathrm{mg} \mathrm{EV}$ al inicio del procedimiento y metoclopramida $10 \mathrm{mg}$ EV al finalizar la cirugía. Como 
analgésicos se utilizaron en ambos grupos dipirona $50 \mathrm{mg} / \mathrm{k}$ EV y morfina $0.05 \mathrm{mg} / \mathrm{k} 30$ minutos antes de finalizar la cirugía.

La intensidad del dolor en la UCPA, fue valorada con la EVA 0 a $10(0=$ no dolor, $10=$ peor dolor $)$ en reposo, con intervalos de 30 minutos y el requerimiento de analgésicos fueron registrados en el mismo intervalo de tiempo en nuestra hoja de recolección de datos. Si EVA fue mayor a 5 se administraban $2 \mathrm{mg}$ de morfina. Las náuseas y vómito fueron anotados en número de episodios.

Se valoraba además el Aldrete ${ }^{11}$ de cada paciente cada 15 minutos hasta conseguir un puntaje mayor de 9, y la escala de Bromage con recuperación de la flexión del pie y la pierna ${ }^{12}$ lo cual era indicación para el egreso de la UCPA, junto con una EVA de dolor $<5$. Este tiempo se consideró como recuperación y salida de la UCPA. Se registraron los requerimientos de efedrina y transfusión de hemoderivados, además del grado de satisfacción con la técnica anestésica calificada como: muy buena, buena, regular o mala.

Análisis estadístico: mediante el programa EPI$I N F O$ versión 2002 se realizó el análisis de los datos recogidos y los pareamientos correspondientes a las variables de estudio.

\section{Resultados}

En este estudio con un total de 29 pacientes sometidas a histerectomía abdominal total las características demográficas son similares para el grupo general y los subgrupos obtenidos al separar la población de acuerdo con la técnica anestésica utilizada.

Los promedios fueron: edad 44 años, peso $62,17 \mathrm{~kg}$, tiempo de inducción para $\mathrm{AG}$ 11,75 minutos, para $\mathrm{AR}$ 10,55 minutos, tiempo quirúrgico con AG 105 minutos y AR 106 minutos (Gráficas 1 y 2).

El 10\% de las pacientes sometidas a histerectomía abdominal total bajo AG (dos) requirió manejo far-

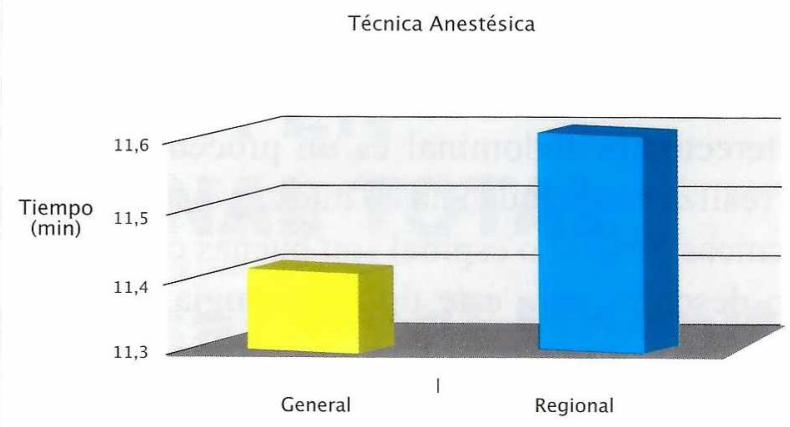

Gráfica I. Tiempo de inducción anestésica según técnica utilizada.

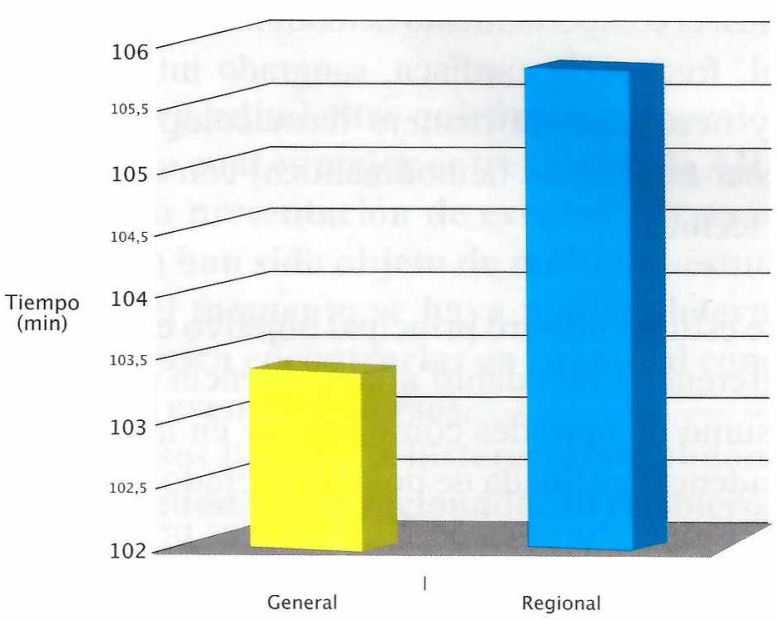

Gráfica 2.Tiempo quirúrgico según técnica utilizada.

macológico con efedrina o vasoactivos para corregir la inestabilidad hemodinámica, mientras en el grupo en el cual se empleó la AR el 11\% (una) necesitó manejo farmacológico.

El promedio de sangrado con $\mathrm{AG}$ fue de $526.75 \mathrm{cc}$, mientras con AR fue de 445.55 cc (Gráfica 3). El uso de hemoderivados de acuerdo con la técnica empleada correspondió a 5\% (una) bajo AG y $1 \%$ (una) con AR.

La valoración del dolor realizada cada treinta minutos mediante la escala visual análoga en el período postoperatorio mostró los siguientes resultados: el promedio a los 30 minutos fue de 6 con AG y 5.4 con AR a los 60 minutos 4.2 y 6.1 , a los 90 minutos 2 y 1.6, a los 150 minutos 0 y 1.1 y por último a los 180 


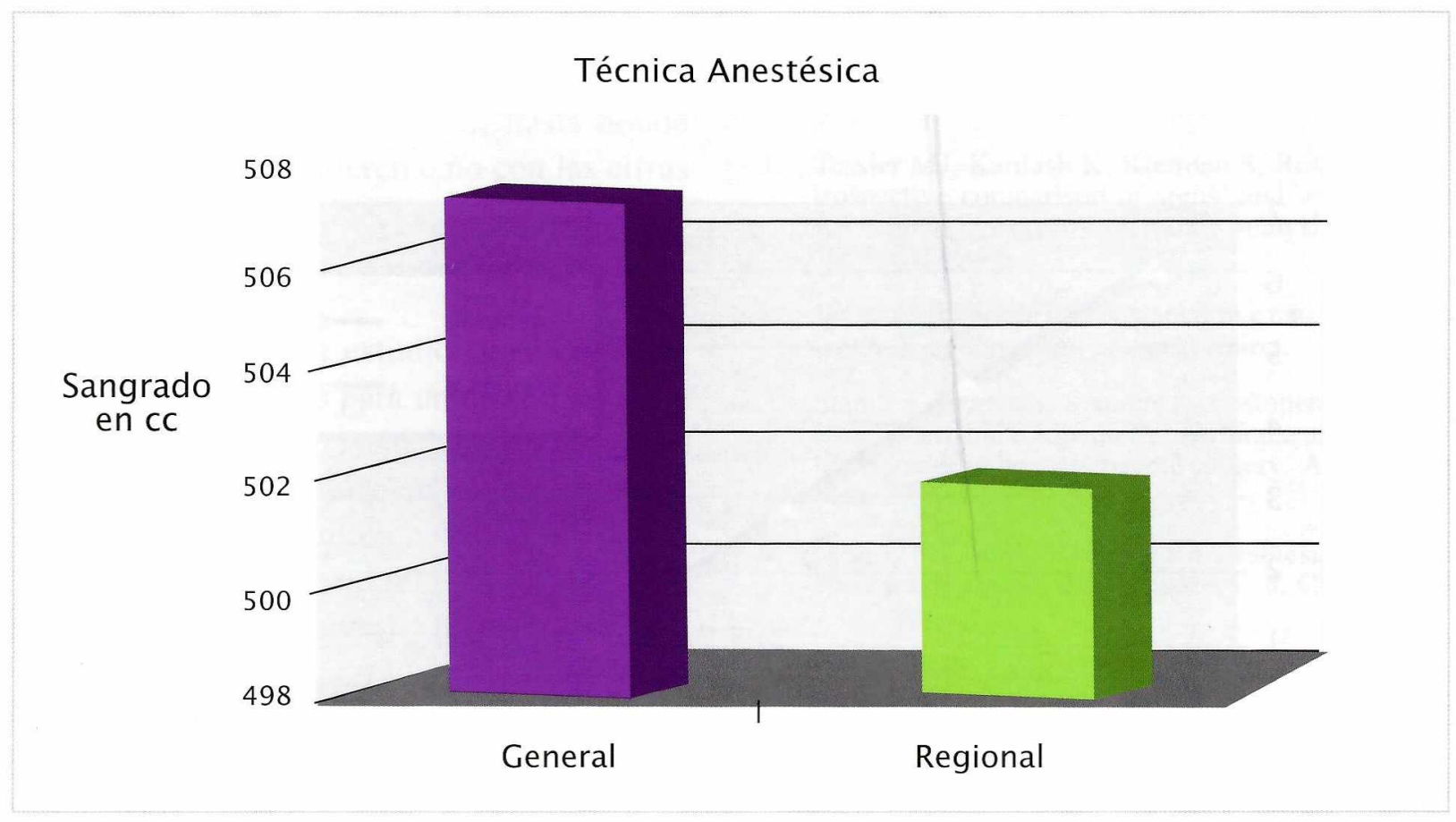

Gráfica 3. Cuantificación total de sangrado intraoperatorio según técnica anestésica.

minutos fue de 0 para ambas técnicas (Gráfica 4). La dosis total de morfina en promedio utilizada en el postoperatorio en miligramos correspondió a $3.9 \mathrm{mg}$ con AG y 4.1 mg AR (Gráfica 5).

La presentación de náuseas en el postoperatorio correspondió a $25 \%$ de las pacientes en las que se utilizó la AG mientras que no hubo ningún caso en el grupo bajo AR. El 20\% con AG presentó vómito en el postoperatorio, mientras que no lo hubo en AR.

La evaluación de la satisfacción de las pacientes con respecto a la técnica, reveló que el $20 \%$ en quienes se empleó AG la calificaron como muy buena, el 75\% buena y el 5\% regular; para la AR el 22\% la calificó como muy buena, $77 \%$ buena y no hubo calificación regular o mala.

\section{Discusión}

De acuerdo con los resultados obtenidos en el presente estudio y las apreciaciones de la literatura sobre cuál de las dos técnicas resulta más efectiva para control de dolor postoperatorio y eventos adversos, así como sangrado y requerimiento de transfusiones, no nos permiten establecer una tendencia clara que pueda considerar superior a alguna. Iniciando con el dolor postoperatorio, los promedios para ambas técnicas son similares a lo largo del tiempo comprendido entre el ingreso de las pacientes a recuperación y la salida a los 180 minutos, lo que nos permite afirmar que ambas son efectivas, contrario a los estudios de Standl T. y Pain Clinic, St Richard's Hospital. ${ }^{6}$ Los requerimientos de morfina durante la estancia en el postoperatorio también fueron similares para ambas técnicas y los tiempos de inducción anestésica y quirúrgico no presentan diferencias significativas, desmitificando la creencia de muchos cirujanos sobre el tiempo de inducción prolongado con AR.

Así mismo, el tiempo quirúrgico resulta de importancia cuando la anestesia interfiere con el desarrollo normal de la técnica quirúrgica, bien sea con AG cuando un plano anestésico inadecuado puede generar dificultad para visualizar o ampliar el campo quirúrgico y la manipulación de las estructuras anatómicas relacionadas, o durante $\mathrm{AR}$, cuando un nivel sensitivo o motor inadecuados puede ocasionar el mismo tipo de situaciones, sumadas al malestar 


\section{Valoración del dolor en la UCPA}

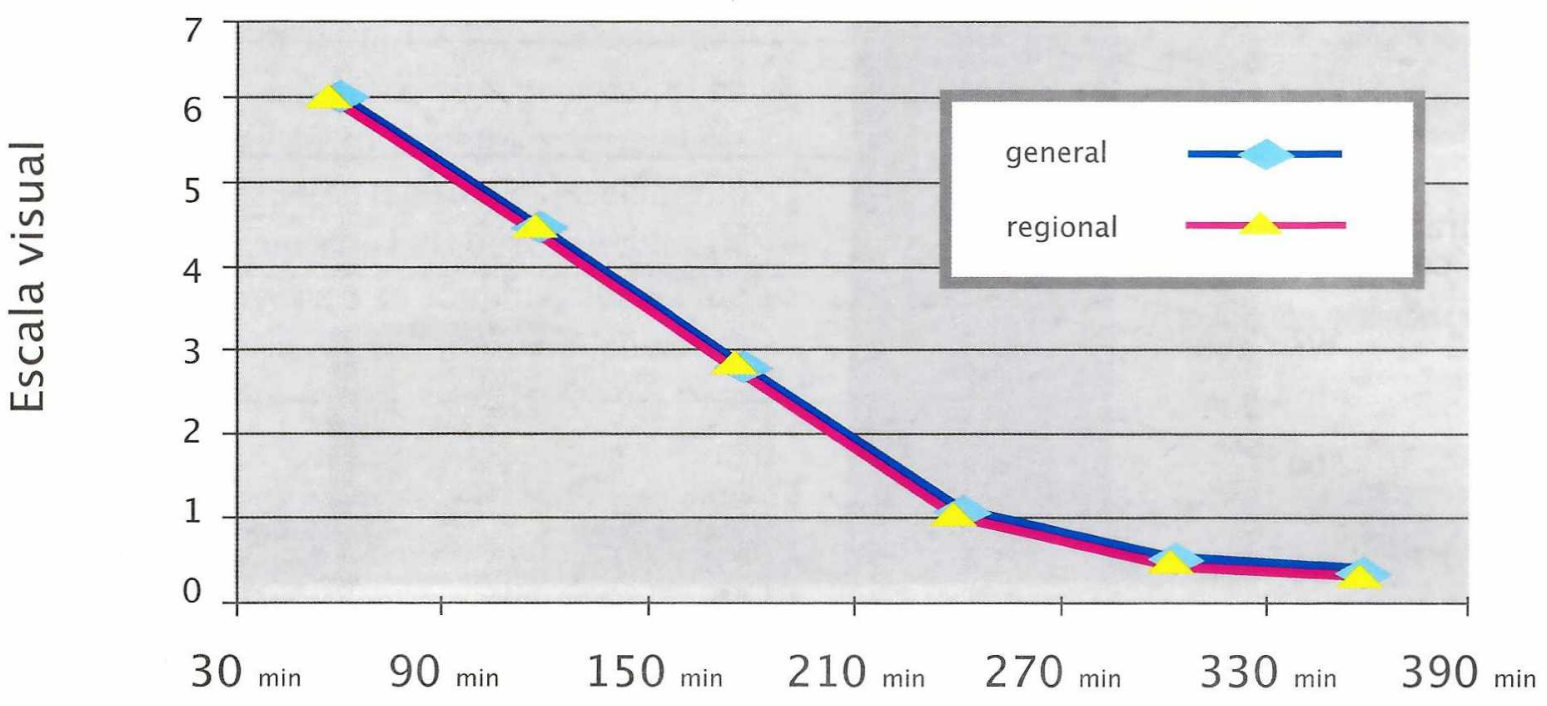

Gráfica 4. Tiempo en minutos

de la paciente.

Quizás con respecto al sangrado valga la observación de una tendencia a la disminución durante el transoperatorio en las pacientes en las cuales se empleó la AR, lo que concuerda con las observaciones realizadas por Tessler MJ, Kardash K, Kleiman S y Rossignol M. ${ }^{1}$ Lo que se traduce en menor número de transfusiones con hemoderivados.

Los eventos en los cuales observamos diferencia significativa entre las dos técnicas corresponden a la presentación de nauseas y vómito postoperatorio, al no presentarse con AR, lo que concuerda con los estudios y observaciones realizadas por Standl T y Watcha MF. ${ }^{2}$

En cuanto a la estabilidad hemodinámica, tampoco se observan diferencias significativas en el requerimiento de fármacos vasoactivos entre las dos técnicas anestésicas.

En relación con la evaluación de la satisfacción de las pacientes, no existen en la literatura datos que

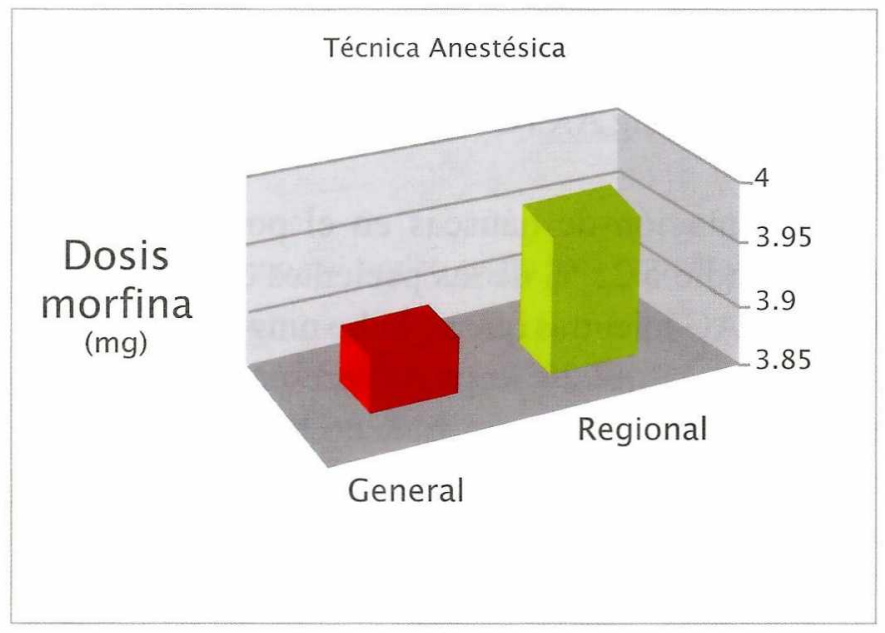

Gráfica 5. Dosis total de morfina requerida en la UCPA según técnica

soporten evidencia significativa sobre esta variable. Lo que observamos en el presente estudio es una mayor satisfacción entre quienes se empleó AR.

En resumen, con el limitado número en nuestra casuística podemos decir que la AR brinda una analgesia similar a la $\mathrm{AG}$ en el período postoperatorio temprano. Las ventajas de la AR corresponden a la presentación de menor sangrado transoperatorio y 
baja incidencia de náuseas y vómito. Se debe realizar el estudio correspondiente con una muestra representativa para poder determinar hasta donde los resultados obtenidos difieren o no con las cifras establecidas por investigaciones previas y las revisiones sistemáticas.

Limitaciones: el presente estudio tiene como limitaciones las establecidas para un diseño de corte transversal, por lo tanto los resultados obtenidos solo son aplicables para esta población en particular; de igual forma la ausencia de diferencias significativas en la presentación de las variables aquí descritas puede corresponder a una muestra no representativa y por lo tanto se requieren estudios para poder determinar fuerza de asociación y demás parámetros estadísticos que permitan demostrar la hipótesis de manera adecuada y precisa.

\section{Conclusión}

En el presente estudio no se observan diferencias significativas en cuanto a dolor postoperatorio, requerimiento de opioides en el período postoperatorio, tiempos de inducción y quirúrgico y estabilidad hemodinámica entre las dos técnicas aquí descritas.

Se observa diferencia significativa en la presentación de eventos adversos como náuseas y vómito postoperatorio en el grupo de pacientes sometidas a AG así como menor sangrado y requerimiento de transfusión de hemoderivados en el grupo con AR. La satisfacción de las pacientes en general es buena con cualquier técnica utilizada y no hubo mala calificación para ninguna de las dos.

Por último, el uso de una u otra técnica para la histerectomía abdominal total sigue a criterio del anestesiólogo, el conocimiento del procedimiento, la habilidad y la experiencia del mismo, dirigidos a un solo objetivo que corresponde al bienestar y la seguridad de las pacientes.

\section{Referencias}

1. Tessler MJ, Kardash K, Kleiman S, Rossignol M. A retrospective comparison of spinal and general anesthesia for vaginal hysterectomy: a time analysis. Anesth Analg 1995; 81: 694-6.

2. Watcha MF, White PF. Postoperative nausea and vomiting. Its etiology, treatment and prevention.

3. Standl T, Eckert S, Schulte J. Postoperative complaints after spinal and thiopentone-isoflurane anaesthesia in patients undergoing orthopaedic surgery. Acta Anaesthesiol Scan 1996; 40: 222-6.

4. Mulroy MF, Willis RP. Spinal anaesthesia for outpatients: appropriate agents and techniques. J. Clin Anesth 1995; 7:622-7.

5. Derville MT, Lang CE. Time to discharge after general versus regional anaesthesia Br. J. Anaesth 1997, 78(Supp I) A3 (abstract).

6. Pain Clinic, St Richard's Hospital. Effectiveness of acute postoperative pain management: Evidence from published data.

7. Audit Commission. Anaesthesia under examination. Audit Commission, London, 1997. Carlsson P. Spinal anaesthesia in young patients using a 29 gauge needle: technical considerations and an evaluation of postoperative complaints compared with general anaesthesia. Br. J Anaesth 1990; 64: 178-82.

8. Wong J, FRCPC. Apinal anesthesia improves the early recovery profile of patients undergoing ambulatory knee arthroscopy. Canadian Journal of Anesthesia 48: 369374.

9. T.Callesen, L.Schouenborg, D. Nielsen, H. Guldager and $\mathrm{H}$, Kehlet. Combined espidural - spinal opiod-free anaesthesia and Analgesia for hysterectomy. British Journal of Anaesthesia. 82 (6) 881-5. 1999.

10. Thoren T, Sundberg A, Wattwill M, Garvill J-E. Jugensen U. Effects of epidural bupivacaine and epidural morphine on bowel function and pain after hysterectomy. Acta Anaesthesiol Scan 1989; 33: 181-5.

11. Rickford JK, Speedy HM, Tytler JA, Lim M. Comparative evaluation of general, epidural ans spinal anaesthesia for extracorporeal shockwave lithotripsy. Ann R Coll Surg Engl 1988; 70: 69-73.

12. Aldrete JA, Kroulik D. A postanesthetic recovery score. Anesthe Analg 1970; 49: 924-34. 